\title{
Validation of the Publication of New Names and New Combinations Previously Effectively Published Outside the IJSB
}

\author{
List No. $51 \dagger$
}

The purpose of this announcement is to effect the valid publication of the following new names and new combinations under the procedure described previously [Int. J. Syst. Bacteriol. 27(3):iv, 1977]. Authors and other individuals wishing to have new names and/or combinations included in future lists should send the pertinent reprint or a photocopy thereof to the IJSB (c/o ASM) for confirmation that all of the other requirements for valid publication have been met. It should be noted that the date of valid publication of these new names and combinations is the date of publication of this list, not the date of the original publication of the names and combinations. The authors of the new names and combinations are as given below, and these authors' names will be included in the author index of the present issue and in the volume author index in this issue of the IJSB.

Inclusion of a name on these lists validates the name and thereby makes it available in bacteriological nomenclature. The inclusion of a name on this list is not to be construed as taxonomic acceptance of the taxon to which the name is applied. Indeed, some of these names may, in time, be shown to be synonyms, or the organisms may be transferred to another genus, thus necessitating the creation of a new combination.

\begin{tabular}{|c|c|c|c|c|}
\hline Name & Proposed as: & Author(s) (reference) & Priority $^{a}$ & Nomenclatural type ${ }^{b}$ \\
\hline Brevibacterium mcbrellneri & New species & McBride et al. (4) & 4 & Strain E2cr (= ATCC 49030) \\
\hline Catenococcus & New genus & Sorokin (5) & 2 & Catenococcus thiocyclus \\
\hline Catenococcus thiocyclus & New species & Sorokin (5) & 2 & $\begin{array}{l}\text { Strain TG 5-3 (= LMD } 92.12=\text { DSM } \\
\quad 9165=\text { ATCC 51228) }\end{array}$ \\
\hline Neisseria iguanae & New species & Barrett et al. (2) & 1 & Strain NVSL 85737 ( = ATCC 51483) \\
\hline Paenibacillus & New genus & Ash et al. (1) & 1 & Paenibacillus polymyxa \\
\hline $\begin{array}{l}\text { Paenibacillus polymyxa (basonym: } \\
\text { Bacillus polymyxa) }\end{array}$ & New combination & Ash et al. (1) & 1 & ATCC 842 \\
\hline $\begin{array}{l}\text { Rhodobacter blastica (basonym: } \\
\text { Rhodopseudomonas blastica) }\end{array}$ & New combination & Kawasaki et al. (3) & 3 & NCIMB $11576=$ ATCC 33485 \\
\hline
\end{tabular}

$\dagger$ Lists 1 through 50 were published in the Int. J. Syst. Bacteriol. 27:306, 1977; 29:79, 436, 1979; 30:601, 676, 1980; 31:215, 382, 1981; 32:266, 384, 1982; 33:438, 672, 896,$1983 ; \mathbf{3 4}: 91,270,355,503,1984 ; \mathbf{3 5}: 223,375,535,1985 ; \mathbf{3 6}: 354,489, \mathbf{5 7 3}, 1986 ; \mathbf{3} 7: 179,1987 ; \mathbf{3 8 : 1 3 6}, 220,328,449,1988 ; \mathbf{3 9}: 93,205,371,495,1989 ; \mathbf{4 0}: 105,212$, $320,470,1990 ; 41: 178,331,456,580,1991 ; 42: 191,327,511,656,1992 ; 43: 188,398,624,864,1993 ;$ and 44:182, $370,595,1994$

a Priority number assigned according to the date the documentation and request for validation are received.

${ }^{b}$ Abbreviations: ATCC, American Type Culture Collection, Rockville, Md.; DSM, Deutsche Sammlung von Mikroorganismen und Zellkulturen GmbH, Braunschweig, Federal Republic of Germany; LMD, Delft University of Technology, Delft, The Netherlands; NCIMB, National Collection of Industrial and Marine Bacteria, Ltd., Aberdeen, Scotland.

\section{REFERENCES}

1. Ash, C., F. G. Priest, and M. D. Collins. 1993. Molecular identification of rRNA group 3 bacilli (Ash, Farrow, Wallbanks and Collins) using a PCR probe test. Antonie van Leeuwenhoek 64:253-260.

2. Barrett, S. J., L. K. Schlater, R. J. Montali, and P. H. A. Sneath. 1994. A new species of Neisseria from iguanid lizards, Neisseria iguanae sp. nov. Lett. Appl. Microbiol. 18:200-202.

3. Kawasaki, H., Y. Hoshino, A. Hirata, and K. Yamasato. 1993. Is intracytoplasmic membrane structure a generic criterion? It does not coincide with phylogenetic interrelationships among phototrophic purple nonsulfur bacteria. Arch. Microbiol. 160:358-362.

4. McBride, M. E., K. M. Ellner, H. S. Black, J. E. Clarridge, and J. E. Wolf. 1993. A new Brevibacterium sp. isolated from infected genital hair of patients with white piedra. J. Med. Microbiol. 39:225-261.

5. Sorokin, D. Y. 1992. Catenococcus thiocyclus gen. nov. sp. nov.-a new facultatively anaerobic bacterium from a near-shore sulphidic hydrothermal area. J. Gen. Microbiol. 138:2287-2292. 